\title{
ANÁLISE DE CONTROLE DE DOPING POR ACETAZOLAMIDA UTILIZANDO O SENSOR ELETROQUÍMICO BASEADO NO $\operatorname{EPCM}-\left[\mathrm{Mn}^{\mathrm{III} / \mathrm{IV}}(\mu-\mathrm{O})(\text { phen })_{2}\right]_{2}\left(\mathrm{ClO}_{4}\right)_{3}$
}

\author{
DOPING CONTROL ANALYSIS BY ACETAZOLAMIDE USING THE \\ ELECTROCHEMICAL SENSOR BASED ON MCPE- $\left[\mathrm{Mn}^{111 / 1 \mathrm{~V}}(\mu-\right.$ \\ O)(phen $\left.)_{2}\right]_{2}\left(\mathrm{ClO}_{4}\right)_{3}$
}

Wesley Bruno da Silva Machini, Marcos Fernando de Souza Teixeira

Universidade Estadual Paulista, Faculdade de Ciências e Tecnologia, Departamento de Física, Química e Biologia, Laboratório de Pesquisa em Eletroanalítica e Sensores, Presidente Prudente/SP, FAPESP. E-mail: wesley machini@hotmail.com, funcao@fct.unesp.br

RESUMO - A preparação e caracterização eletroquímica de sensor eletroquímico baseado no eletrodo de pasta de carbono modificado com complexo $\left[\mathrm{Mn}^{\mathrm{III} / \mathrm{IV}}(\mu-\mathrm{O})(\text { phen })_{2}\right]_{2}\left(\mathrm{ClO}_{4}\right)_{3}$, bem como sua aplicação na detecção de acetazolamida com interesse analítico no controle de doping são relatados. O comportamento eletroquímico do sensor e a eletrooxidação de ACTZ foram investigadas utilizando a técnica de voltametria cíclica e de varredura linear, respectivamente. O voltamograma cíclico para o sensor eletroquímico apresentou um processo redox correspondente ao par $\mathrm{Mn}^{\mathrm{III} / \mathrm{IV}} / \mathrm{Mn}^{\mathrm{IV} / \mathrm{IV}}$ e propriedades eletrocatalíticas na oxidação de ACTZ. A resposta voltamétrica do sensor foi obtida em um intervalo de concentração de $5,00 \times 10^{-7} \mathrm{~mol}$ $\mathrm{L}^{-1}$ a $5,00 \times 10^{-6} \mathrm{~mol} \mathrm{~L}^{-1}$ sendo a resposta linear entre $5,00 \times 10^{-7} \mathrm{~mol} \mathrm{~L}^{-1}$ e $3,50 \times 10^{-6} \mathrm{~mol} \mathrm{~L}^{-1}$ com limite de quantificação de $9,90 \times 10^{-5} \mathrm{~mol} \mathrm{~L}^{-1}$, limite de detecção de $2,97 \times 10^{-6} \mathrm{~mol} \mathrm{~L}^{-1}$ e sensibilidade de $39,29 \mu \mathrm{A} \mathrm{L}$ $\mathrm{mol}^{-1}$.

Palavras-chave: acetazolamida; doping; sensor eletroquímico.

Recebido em: 21/08/2014

Revisado em: 08/09/2014_ Aprovado em: 08/09/2014
ABSTRACT - The preparation and electrochemical characterization of an electrochemical sensor based on carbon paste electrode modified with $\left[\mathrm{Mn}^{\mathrm{III} / \mathrm{IV}}(\mu-\mathrm{O})(\text { phen })_{2}\right]_{2}\left(\mathrm{ClO}_{4}\right)_{3}$, as well as its application in detection of acetazolamide with interest in doping control analysis are related. The electrochemical behavior of the sensor and the electrooxidation of ACTZ were investigated using the technique of cyclic and linear sweep voltammetry, respectively. The cyclic voltammogram for the electrochemical sensor showed one redox process assigned to $\mathrm{Mn}^{\mathrm{III} / \mathrm{IV}} / \mathrm{Mn}^{\mathrm{IV} / \mathrm{IV}}$ couple and electrocatalytic properties in oxidation of ACTZ. The voltammetric response was obtained in a concentration range from $5.00 \times 10^{-7} \mathrm{~mol} \mathrm{~L}^{-1}$ to $5.00 \times 10^{-6} \mathrm{~mol} \mathrm{~L}^{-1}$, being the linear response from $5.00 \times 10^{-7} \mathrm{~mol} \mathrm{~L}^{-1}$ to $3.50 \times 10^{-6} \mathrm{~mol} \mathrm{~L}^{-1}$ with limit of quantification $9.90 \times 10^{-5} \mathrm{~mol} \mathrm{~L}^{-1}$, limit of detection $2.97 \times 10^{-6} \mathrm{~mol} \mathrm{~L}^{-1} \mathrm{e}$ sensitivity of $39.29 \mu \mathrm{A} \mathrm{L} \mathrm{mol}{ }^{-1}$.

Keywords: acetazolamide; doping; electrochemical sensor. 


\section{INTRODUÇÃO}

O advento do uso de substâncias que melhoram o desempenho de atletas para dar-lhes vantagem competitiva, seja como "drogas" ou compostos altamente purificados e potentes, acabou resultando na triagem sistemática para detectar e impedir o uso de tais substâncias, comumente referido como "doping" (FRASER, 2004). A lista de substâncias, supervisionada pela Agência Mundial Antidoping (WADA), inclui atualmente agentes anabolizantes, hormônios e substâncias relacionadas, betaagonistas, agentes antiestrogênicos, diuréticos e outros agentes mascarantes como proibidos.

A acetazolamida (ACTZ), N-[5(Aminosulfonil)-1,3,4-tiadiazol-2-il]acetamida (Figura 1), é um inibidor da enzima anidrase carbônica usada clinicamente no tratamento de glaucoma (KAUR et al., 2002). Também, é utilizada sozinha ou em associação com medicamentos antiepiléticos para 0 tratamento de várias formas de epilepsia (REISS; OLES, 1996) e ainda, como fármaco para a profilaxia de doenças de alta altitude (LOW et al., 2012), hipertensão intracraniana idiopática (WALL et al., 2014), cistinúria, paralisia periódica (BRAYFIELD, 2014), apnéia do sono central (AURORA et al., 2014) e ectasia dural.
Segundo a Agência Mundial Antidoping, a ACTZ é uma substância proibida dentro e fora de competição, já que por agir como um diurético pode ser considerada como um agente "ocultador ou camuflador" de drogas ilegais específicas procuradas nos testes de doping. Ainda que proibidos em competições, os diuréticos são utilizados para reduzir rapidamente o peso corporal em esportes em que há categorias de pesos e também como tentativa de aumentar a excreção urinária e assim eliminar mais rapidamente eventuais substâncias dopantes.

Muitas técnicas de detecção de ACTZ em amostras biológicas têm sido desenvolvidas: biossensores ópticos de matriz sol-gel (JERONIMO et al., 2005), cromatografia líquida de gás com detector de captura de elétrons (WALLACE; SHAH; RIEGELMAN, 1977), cromatografia líquida de alta eficiência (CHAPRON; WHITE, 1984; HARTLEY et al., 1986; ICHIKAWA et al., 1998; ZARGHI; SHAFAATI, 2002), ensaios enzimáticos (GREENE; KENNY, 1992) e polarografia (DE BALUGERA, GOICOLEA; BARRIO, 1994).

No entanto, a maioria dos métodos citados requer várias e demoradas etapas de manipulação, instrumentos sofisticados e treinamento especial. Surge então a possibilidade de utilização de sensores eletroquímicos aplicados à detecção de ACTZ, 
já que estes têm sido pouco estudados (GHOLIVAND; PARVIN, 2011; KHODADADIAN; AHMADI, 2010; SHAKIBAIAN; PARVIN, 2012). Dessa forma, objetivou-se através deste trabalho construir e caracterizar eletroquimicamente um eletrodo de pasta de carbono modificado com complexo bis( $\mu$ oxo)dimanganês(III,IV)-tetraquis-1,10-

fenantrolina com intuito de aplicá-lo como um sensor eletroquímico na detecção de acetazolamida com interesse analítico no controle de doping.<smiles>CC(=O)Nc1nnc(S(N)(=O)=O)s1</smiles>

Figura 1. Representação estrutural do $N$ [5(aminosulfonil)-1,3,4-tiadiazol-2-il]acetamida (ACTZ).

\section{METODOLOGIA}

\subsection{SÍNTESE DO COMPLEXO BIS( $\mu$-OXO)- DIMANGANÊS(III,IV)-TETRAQUIS-1,10- FENANTROLINA}

A síntese do complexo metálico $\left[\mathrm{Mn}^{\text {III/IV }}(\mu-\mathrm{O})(\text { phen })_{2}\right]_{2}\left(\mathrm{ClO}_{4}\right)_{3}$ foi realizada de acordo com adaptações de um procedimento citado na literatura (COOPER; CALVIN, 1977). Uma solução de $1,08 \mathrm{~g}$ de $\mathrm{Mn}\left(\mathrm{CH}_{3} \mathrm{COO}\right)_{2} \cdot 4 \mathrm{H}_{2} \mathrm{O}(4,4 \mathrm{mmol})$ em $15 \mathrm{~mL}$ de água deionizada foi adicionada à $2,37 \mathrm{~g}$ de 1,10-fenantrolina (13 mmol) em 7,5 mL de acetona, formando uma solução amarela. A esta solução foram adicionados $20 \mathrm{~mL}$ de tampão acetato $1,0 \mathrm{~mol} \mathrm{~L}^{-1}(\mathrm{pH} \mathrm{4,5)} \mathrm{e} \mathrm{o} \mathrm{pH}$ ajustado com ácido acético glacial à 4,5. Após, a solução resultante foi resfriada em banho de gelo até $0{ }^{\circ} \mathrm{C}$ e $0,30 \mathrm{~g}$ de $\mathrm{KMnO}_{4}$ (1,9 mmol) em $12,5 \mathrm{~mL}$ de água destilada foram adicionados gota à gota sob agitação constante. Uma solução verde foi formada e esta foi agitada por 15 minutos a $0{ }^{\circ} \mathrm{C}$. Ao final do tempo, uma solução de $\mathrm{NaClO}_{4}$ 1,0 mol $L^{-1}$ foi adicionada e o precipitado formado foi filtrado, lavado com etanol e seco à temperatura ambiente.

\subsection{CONSTRUÇÃO DO SENSOR ELETROQUÍMICO}

O sensor eletroquímico baseado no eletrodo de pasta de carbono modificado com complexo $\left[\mathrm{Mn}^{\mathrm{III} / \mathrm{IV}}(\mu-\mathrm{O})(\text { phen })_{2}\right]_{2}\left(\mathrm{ClO}_{4}\right)_{3}$ foi preparado pela mistura de $60 \%(\mathrm{~m} / \mathrm{m})$ de pó de grafite, $30 \%(\mathrm{~m} / \mathrm{m})$ de aglutinante (óleo mineral) e $10 \%(\mathrm{~m} / \mathrm{m})$ do complexo metálico bis( $\mu$-oxo)dimanganês(III,IV)-tetraquis-1,10-

fenantrolina. Nesta mistura foram adicionados $20 \mathrm{~mL}$ de hexano e então colocada sobre agitação em um agitador magnético até a completa evaporação do solvente. Este compósito foi colocado no interior de uma das extremidades de um tudo de Teflon $^{\circledR}$ (diâmetro interno $=0,8 \mathrm{~cm}$, diâmetro externo $=1,0 \mathrm{~cm}$ e comprimento $=$ $15 \mathrm{~cm}$ ) e então compactada pressionando-se uma haste de aço inoxidável (diâmetro =0,8 $\mathrm{cm}$ e comprimento $=20 \mathrm{~cm})$, colocada na 
outra extremidade do tubo contra uma superfície plana e inerte.

\subsection{COMPORTAMENTO ELETROQUÍMICO DO SENSOR}

O comportamento eletroquímico do sensor baseado no eletrodo de pasta de carbono modificado com complexo bis( $\mu$ oxo)dimanganês(III,IV)-tetraquis-1,10-fenantrolina foi investigado por voltametria cíclica em tampão acetato $0,1 \mathrm{~mol} \mathrm{~L}^{-1}(\mathrm{pH} \mathrm{4,76)}$.

\subsection{DESEMPENHO DO SENSOR ELETROQUÍMICO PARA ACETAZOLAMIDA}

Inicialmente, a atividade eletrocatalítica do sensor na eletrooxidação de ACTZ foi investigada utilizando a técnica de voltametria cíclica em um intervalo de potencial de 0,0 a $1,2 \mathrm{~V}$ vs. ECS com velocidade de varredura de $25 \mathrm{mV} \mathrm{s}^{-1}$. Por fim, a determinação de ACTZ foi realizada pela aplicação de potenciais utilizando voltametria de varredura linear em um intervalo de potencial de 0,35 a 1,10 V vs. ECS com velocidade de varredura de $25 \mathrm{mV} \mathrm{s}^{-}$ ${ }^{1}$. O sensor eletroquímico foi submetido a varreduras de potenciais em $20 \mathrm{~mL}$ de tampão acetato $0,1 \mathrm{~mol} \mathrm{~L}^{-1}(\mathrm{pH} 5,6)$. A avaliação do desempenho do sensor para ACTZ foi conduzida pela adição do analito (1 $\mathrm{mmol} \mathrm{L^{-1 }}$ ) na célula eletroquímica.

\section{RESULTADOS}

3.1 COMPORTAMENTO ELETROQUÍMICO DO SENSOR EM SOLUÇÃO AQUOSA
Com a finalidade de compreender os mecanismos eletroquímicos do sensor eletroquímico para posterior aplicação na determinação de ACTZ, voltamogramas cíclicos em solução aquosa foram registrados. A Figura 2 apresenta o voltamograma, o qual revelou um sistema quasi-reversível com um processo redox $\left(E_{\mathrm{pa}}=0,795 \mathrm{~V}\right.$ e $E_{\mathrm{pc}}=0,444 \mathrm{~V}$ vs. ECS) com uma variação de potencial $\left(\Delta E_{\mathrm{p}}\right)$ de $0,351 \mathrm{~V} v s$. ECS e potencial de meia $\left(E_{\mathrm{p} / 2}\right)$ de 0,620 V vs. ECS.

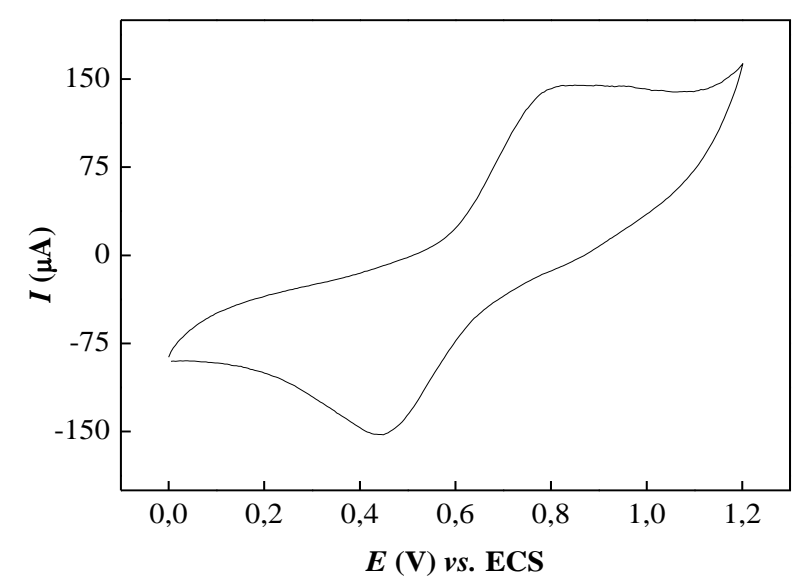

Figura 2. Voltamograma cíclico para sensor eletroquímico em tampão acetato $0,1 \mathrm{~mol} \mathrm{~L}^{-1}$ (pH 4,76) utilizando um intervalo de potencial de 0,0 a 1,2 (V) vs. ECS com velocidade de varredura de $25 \mathrm{mV} \mathrm{s}^{-1}$.

\subsection{DETERMINAÇÃO DE ACETAZOLAMIDA UTILIZANDO O SENSOR ELETROQUÍMICO}

A atividade catalítica do sensor eletroquímico na eletrooxidação de ACTZ é apresentada na Figura 3. Nesta, é possível observar o incremento de corrente de pico anódica e catódica com o aumento da concentração de ACTZ. 


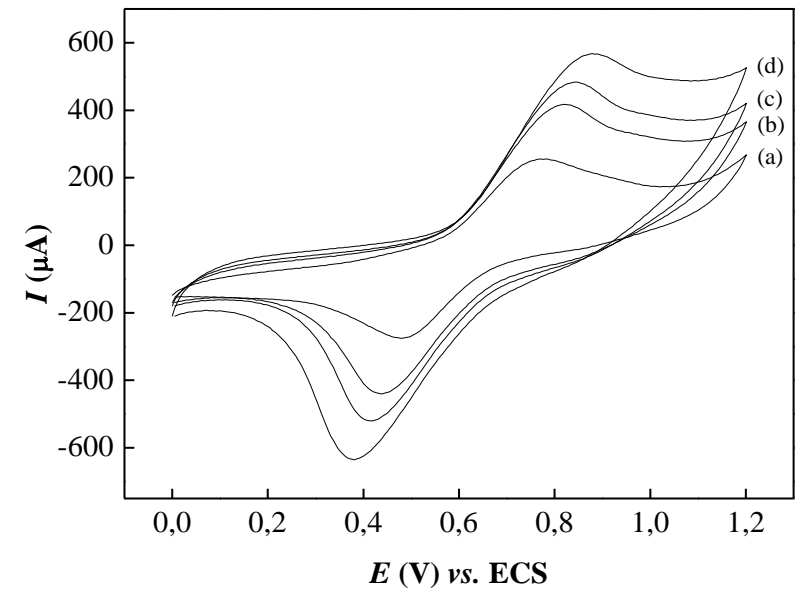

Figura 3. Voltamogramas cíclicos para 0 sensor eletroquímico em tampão acetato 0,1 $\mathrm{mol} \mathrm{L}^{-1}(\mathrm{pH} 4,76)$ na (a) ausência e presença de (b) $1,00 \times 10^{-6} \mathrm{~mol} \mathrm{~L}^{-1}$, (c) $2,50 \times 10^{-6} \mathrm{~mol}$ $\mathrm{L}^{-1}$ e (d) 5,00 $\times 10^{-6} \mathrm{~mol} \mathrm{~L}^{-1}$ de ACTZ utilizando um intervalo de potencial de 0,0 a 1,2 (V) vs. ECS com velocidade de varredura de $25 \mathrm{mV}$ $s^{-1}$.

\section{Demonstrada a atividade} eletrocatalítica, medidas voltamétricas de varredura linear foram realizadas em um intervalo de potencial de 0,35 a $1,10 \mathrm{~V}$ vs. ECS em diferentes concentrações de ACTZ a fim de obter uma curva analítica. Os voltamogramas lineares foram obtidos em tampão acetato $(\mathrm{pH} 5,6)$ variando a contração de $5,00 \times 10^{-7} \mathrm{~mol} \mathrm{~L}^{-1}$ a 5,00 × $10^{-6}$ $\mathrm{mol} \mathrm{L}^{-1}$ de ACTZ (Figura 4).

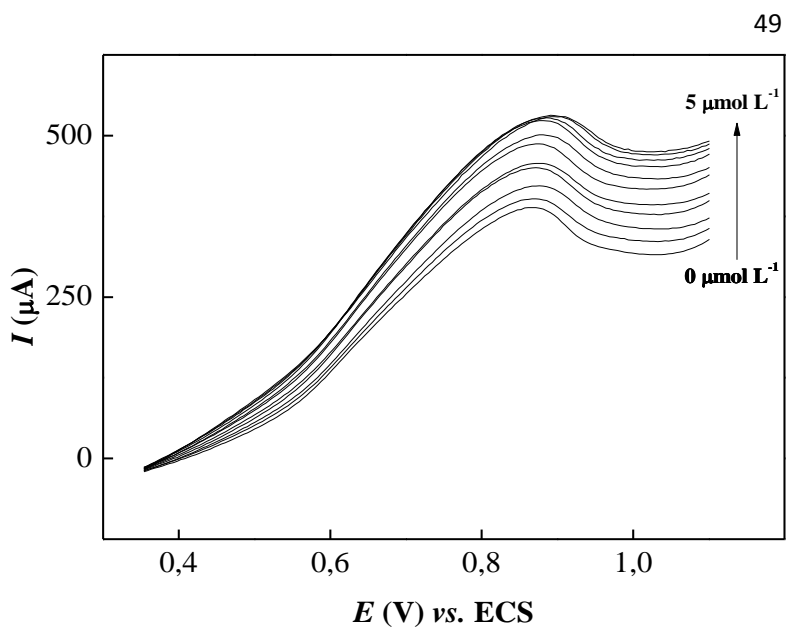

Figura 4. Voltamogramas lineares para o sensor eletroquímico em tampão acetato 0,1 $\mathrm{mol} \mathrm{L}^{-1}(\mathrm{pH} 5,6)$ na ausência e com sucessivas adições de ACTZ utilizando um intervalo de potencial de 0,35 a 1,10 (V) vs. ECS com velocidade de varredura de $25 \mathrm{mV} \mathrm{s}^{-1}$.

A Figura 5 apresenta a variação da corrente de pico anódica em função da concentração de ACTZ, onde se obteve um intervalo linear de concentração de 5,00 × 10${ }^{7} \mathrm{~mol} \mathrm{~L}^{-1}$ a $3,50 \times 10^{-6} \mathrm{~mol} \mathrm{~L}^{-1}$ com limite de quantificação (dez vezes o sinal do branco/coeficiente angular) de 9,90 × $10^{-5}$ mol L ${ }^{-1}$, limite de detecção (três vezes o sinal do branco/coeficiente angular) de 2,97 $\times 10^{-6}$ $\mathrm{mol} \mathrm{L}^{-1}$ e sensibilidade $39,29 \mu \mathrm{A} \mathrm{L} \mathrm{mol}{ }^{-1}$ sendo a equação de regressão linear:

$$
\begin{array}{r}
I_{\text {pa }}(A)=39,29+3,43 \times 10^{-6} \mathrm{~mol} \mathrm{~L}^{-1}[\text { ACTZ }] \\
(n=0,991 \text { e } r=7)
\end{array}
$$




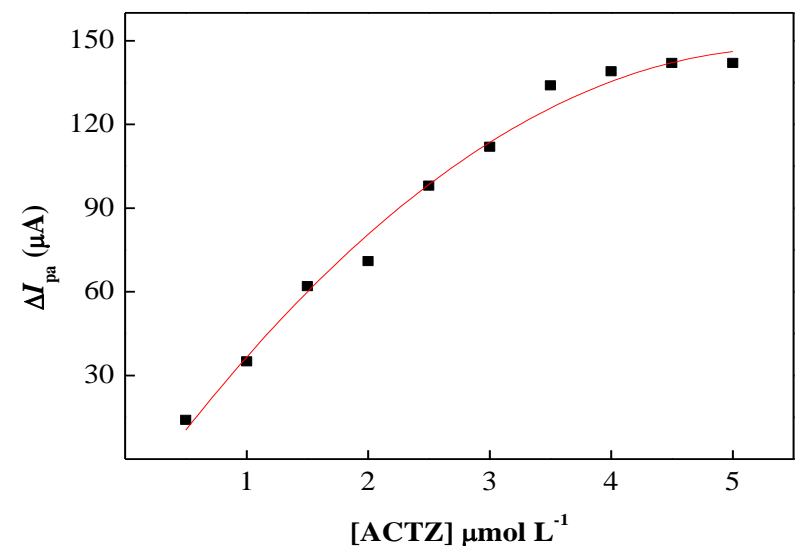

Figura 5. Dependência dos valores de corrente de pico anódica em função da concentração de $5,00 \times 10^{-7} \mathrm{~mol} \mathrm{~L}^{-1}$ a 5,00 $\times$ $10^{-6} \mathrm{~mol} \mathrm{~L}^{-1}$ de ACTZ.

\section{DISCUSSÃO}

O processo redox observado no voltamograma cíclico obtido no estudo do comportamento eletroquímico do sensor (Figura 2) é atribuído ao par redox $M n^{I I I / I V} / \mathrm{Mn}^{\text {IV/IV }}$ conforme a equação:

$\left[\mathrm{Mn}^{\mathrm{III} / \mathrm{IV}}(\mu-\mathrm{O})(\text { phen })_{2}\right]_{2}\left(\mathrm{ClO}_{4}\right)_{3(\mathrm{EPCM})} \rightarrow$ $\left[\mathrm{Mn}^{\mathrm{IV} / \mathrm{IV}}(\mu-\mathrm{O})(\text { phen })_{2}\right]_{2}\left(\mathrm{ClO}_{4}\right)_{3(\mathrm{EPCM})}+1 \mathrm{e}^{-}$

Evidencia-se que, com as sucessivas adições, sensor eletroquímico promove a eletrooxidação de ACTZ através de um mecanismo catalítico onde o analito difundese até a superfície do sensor e os centros metálicos de $\mathrm{Mn}^{\prime \prime \prime} / \mathrm{Mn}^{\mathrm{IV}}$ do complexo são oxidados, ao passo que a ACTZ então oxidada incremente o sinal analítico. No retorno, os centros metálicos de $\mathrm{Mn}^{\mathrm{IV}} / \mathrm{Mn}^{\mathrm{IV}}$ são reduzidos, voltando ao seu estado de oxidação original, perfazendo uma etapa eletroquímica e uma etapa química, de acordo com a seguinte equação:

\section{Etapa eletroquímica:}

$$
2 \mathrm{Mn}^{\mathrm{III}} \mathrm{Mn}^{\mathrm{IV}} \rightarrow 2 \mathrm{Mn}^{\mathrm{IV}} \mathrm{Mn}^{\mathrm{IV}}+2 \mathrm{e}^{-}
$$

\section{Etapa química:}

$2 \mathrm{Mn}^{\mathrm{IV}} \mathrm{Mn}^{\mathrm{IV}}+\mathrm{ACTZ}_{\text {(red) }} \rightarrow$

$$
2 \mathrm{Mn}^{\prime \prime \prime} \mathrm{Mn}^{\mathrm{IV}}+\mathrm{ACTZ}_{(\mathrm{ox})}+2 \mathrm{H}^{+}
$$

Uma tentativa de representação do mecanismo no eletrodo é apresentada na Figura 6.

Através da curva analítica (Figura 5) podem-se determinar e analisar os limites de quantificação $\left(9,90 \times 10^{-5} \mathrm{~mol} \mathrm{~L}^{-1}\right)$ e detecção $\left(2,97 \times 10^{-6} \mathrm{~mol} \mathrm{~L}^{-1}\right)$, e sensibilidade do sensor $\left(39,29 \mu \mathrm{A} \mathrm{L} \mathrm{mol}{ }^{-1}\right)$. Embora o trabalho de Gholivand e Parvin (2011) utilizando um sensor eletroquímico baseado no eletrodo de pasta de carbono modificado com nanopartículas de ouro na determinação de ACTZ apresente um valor de limite de detecção menor $\left(7,1 \mathrm{nmol} \mathrm{L}^{-1}\right)$, os resultados obtidos com o sensor eletroquímico a base de pasta de carbono modificado com complexo bis( $\mu$-oxo)dimanganês(III,IV)-tetraquis-1,10-fenantrolina mostraram-se eficazes na quantificação de ACTZ.

O desvio de linearidade observado para concentrações superiores a 3,50 × $10^{-6}$ $\mathrm{mol} \mathrm{L}^{-1}$ na curva analítica pode ser atribuído à saturação máxima dos sítios eletrocatalíticos 
disponíveis na superfície do sensor, ou seja, na superfície do sensor pode estar uma absorção de ACTZ e subsequentemente ocorrendo.

uma saturação dos sítios ativos de $\mathrm{Mn}^{\prime \prime \prime} / \mathrm{Mn}^{\mathrm{IV}}$

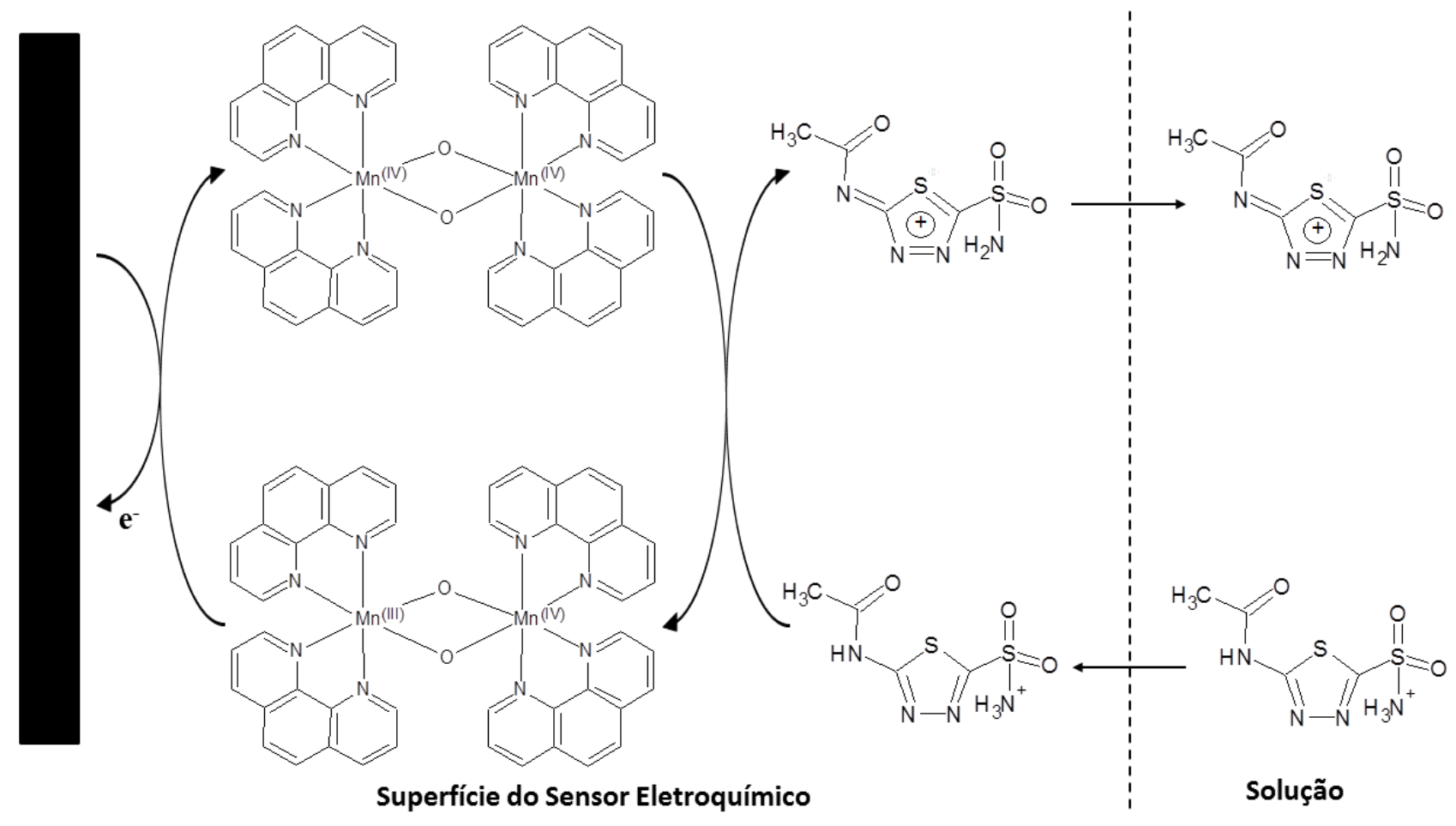

Figura 6. Representação esquemática do mecanismo eletrocatalítico do sensor para ACTZ.

\section{CONSIDERAÇÕES FINAIS}

Este trabalho descreveu um sensor eletroquímico para acetazolamida baseado no eletrodo de pasta de carbono modificado com complexo $\left.\mathrm{Mn}^{\mathrm{III} / \mathrm{IV}}(\mu-\mathrm{O})(\text { phen })_{2}\right]_{2}\left(\mathrm{ClO}_{4}\right)_{3}$. O sensor demonstrou propriedades eletrocatalíticas na eletrooxidação de ACTZ, permitindo a detecção de baixos níveis de concentração com alta sensibilidade do sensor, sugerindo uma promissora plataforma para determinação de ACTZ em amostras biológicas na análise do controle de doping.

\section{AGRADECIMENTOS}

Os autores agradecem a Fundação de Amparo à Pesquisa do Estado de São Paulo FAPESP (2005/01296-4) pelo apoio à pesquisa. A bolsa de estudos concedida pela FAPESP (2013/10469-6) à W. B. S. Machini é imensamente agradecida. Os autores agradecem a SJT.

\section{REFERÊNCIAS}

AURORA, R. N. et al. The Treatment of central sleep apnea syndromes in adults: practice parameters with an evidence-based literature review and meta-analyses. Sleep, v.35, n.1, p.17-40, 2012. http://dx.doi.org/10.5665/sleep.1580 
BRAYFIELD, A. Martindale: The complete drug reference. 38 . ed. Londres: The Pharmaceutical Press, 2014.

CHAPRON, D. J.; WHITE, L. B. Determination of acetazolamide in biological-fluids by reverse-phase high-performance liquidchromatography. Journal of Pharmaceutical Sciences, v.73, n.7, p.985-989, 1984. http://dx.doi.org/10.1002/jps.2600730732

COOPER, S. R.; CALVIN, M. Mixed-valence interactions in $\mathrm{Di}-\mu$-Oxo bridged manganese complexes. Journal of the American Chemical Society, v.99, n.20, p.6623-6630, 1977.

http://dx.doi.org/10.1021/ja00462a025

DE BALUGERA, Z. G.; GOICOLEA, M. A.; BARRIO, R. J. Joint determination of todralazine and acetazolamide in human serum by differential-pulse polarography. Journal of Pharmaceutical and Biomedical Analysis, v.12, n.7, p.883-887, 1994. http://dx.doi.org/10.1016/S0731-

7085(94)80038-3

FRASER, A. D. Doping control from a global and national perspective. Ther Drug Monit, v.26, n.2, p.171-174, 2004. http://dx.doi.org/10.1097/00007691200404000-00015

GHOLIVAND, M. B.; PARVIN, M. H. Voltammetric study of acetazolamide and its determination in human serum and urine using carbon paste electrode modified by gold nanoparticle. Journal of Electroanalytical Chemistry, v.660, n.1, p.163-168, 2011. http://dx.doi.org/10.1016/j.jelechem.2011.0 6.026

GREENE, I. M.; KENNY, A. D. determination of acetazolamide in human serum by enzymatic assay. Journal of Pharmacological and Toxicological Methods, v.28, n.3, p.159-166, 1992. http://dx.doi.org/10.1016/10568719(92)90078-F
HARTLEY, R. et al. Solid-phase extraction of acetazolamide from biological-fluids and subsequent analysis by high-performance liquid-chromatography. Journal of Chromatography, v.377, p.295-305, 1986. http://dx.doi.org/10.1016/S03784347(00)80785-9

ICHIKAWA, N. et al. Quantitation of acetazolamide in rat plasma, brain tissue and cerebrospinal fluid by high-performance liquid chromatography. Journal of Pharmaceutical and Biomedical Analysis, v.17, n.8, p.1415-1421, 1998. http://dx.doi.org/10.1016/S07317085(98)00024-7

JERONIMO, P. C. A. et al. Flow-through sol-gel optical biosensor for the colorimetric determination of acetazolamide. Analyst, v.130, n.8, p.1190-1197, 2005. http://dx.doi.org/10.1039/b504474j

KAUR, I. P. et al. Acetazolamide: future perspective in topical glaucoma therapeutics. International Journal of Pharmaceutics, v.248, n.1-2, p.1-14, 2002. http://dx.doi.org/10.1016/S03785173(02)00438-6

KHODADADIAN, M.; AHMADI, F. Computerassisted design and synthesis of molecularly imprinted polymers for selective extraction of acetazolamide from human plasma prior to its voltammetric determination. Talanta, v.81, n.4-5, p.1446-1453, 2010. http://dx.doi.org/10.1016/j.talanta.2010.02.0 49

LOW, E. V. et al. Identifying the lowest effective dose of acetazolamide for the prophylaxis of acute mountain sickness: systematic review and meta-analysis. British Medical Journal, v.345, 2012. http://dx.doi.org/10.1136/bmj.e6779

REISS, W. G.; OLES, K. S. Acetazolamide in the treatment of seizures. Annals of 
Pharmacotherapy, v.30, n.5, p.514-518, 1996.

SHAKIBAIAN, V.; PARVIN, M. H. Determination of acetazolamide by graphene paste electrode. Journal of Electroanalytical Chemistry, v.683, p.119-124, 2012. http://dx.doi.org/10.1016/j.jelechem.2012.0 7.042

WALL, M. et al. Effect of acetazolamide on visual function in patients with idiopathic intracranial hypertension and mild visual loss: the idiopathic intracranial hypertension treatment trial. Jama-Journal of the American Medical Association, v.311, n.16, p.1641-1651, 2014. http://dx.doi.org/10.1001/jama.2014.3312

WALLACE, S. M.; SHAH, V. P.; RIEGELMAN, S. Glc Analysis of Acetazolamide in Blood, Plasma, and Saliva Following OralAdministration to Normal Subjects. Journal of Pharmaceutical Sciences, v.66, n.4, p.527530, 1977. http://dx.doi.org/10.1002/jps.2600660416

ZARGHI, A.; SHAFAATI, A. Rapid determination of acetazolamide in human plasma. Journal of Pharmaceutical and Biomedical Analysis, v.28, n.1, p.169-172, 2002. http://dx.doi.org/10.1016/S07317085(01)00615-X 